\title{
Pancreatitis-Associated Protein-III Is a Novel Macrophage Chemoattractant Implicated in Nerve Regeneration
}

\author{
Kazuhiko Namikawa, ${ }^{1,2}$ Takashi Okamoto, ${ }^{1}$ Akinobu Suzuki, ${ }^{1}$ Hiroyuki Konishi, ${ }^{1}$ and Hiroshi Kiyama ${ }^{1}$ \\ ${ }^{1}$ Department of Anatomy and Neurobiology, Osaka City University Graduate School of Medicine, Osaka 545-8585, Japan, and ²Department of Functional \\ Anatomy and Neuroscience, Asahikawa Medical College, Asahikawa 078-8510, Japan
}

\begin{abstract}
Circulating macrophages are recruited to degenerating nerves in response to nerve injury to remove myelin and axonal debris, a process that is crucial for successful nerve regeneration. In this study, we demonstrate that pancreatitis-associated protein (PAP)-III is a macrophage chemoattractant that is induced in and released from injured nerves. In vitro experiments revealed that PAP-III possessed a strong macrophage chemoattractant activity that was comparable with that of monocyte chemoattractant protein-1. In addition, gene knockdown via adenovirus-mediated small interference RNA expression in isolated sciatic nerves successfully suppressed PAP-III expression and its macrophage chemoattractant activity. Furthermore, overexpression or knockdown of the PAP-III gene in crushed sciatic nerves in rats resulted in acceleration or retardation of macrophage recruitment and subsequent nerve regeneration, respectively. Collectively, our results demonstrate that PAP-III is a novel macrophage chemoattractant that is involved in peripheral nerve regeneration and further provide new insights into Schwann cell-macrophage interactions and therapeutic interventions.
\end{abstract}

Key words: chemotaxis; macrophage; nerve injury; pancreatitis-associated protein; PAP; regenerating gene; Reg; hepatocarcinomaintestine-pancreas; HIP

\section{Introduction}

Injuries to axons in the peripheral nervous system (PNS) induce the degeneration of distal axons (Wallerian degeneration), a process that is rapidly achieved by both axonal and myelin breakdown (Scherer and Salzer, 2001). Wallerian degeneration is accompanied by orchestrated cellular responses in the injured neurons, dedifferentiated Schwann cells, and cells involved in immune systems (Scherer and Salzer, 2001). Among such cellular responses, the most striking is macrophage invasion into the degenerating nerves, mainly by circulating hematogenous monocytic cells (Beuche and Friede, 1984). Multiple beneficial roles of the invading macrophages appear to contribute to Wallerian degeneration and subsequent axonal regeneration (Hughes and Perry, 2000). These cells effectively scavenge the debris of myelin components that inhibits axonal growth. In addition, they can secrete a number of soluble factors that stimulate axonal growth directly or indirectly.

Although the molecular mechanisms underlying the macro-

Received Jan. 4, 2006; revised June 11, 2006; accepted June 12, 2006.

We thank Dr. S. Yoshida (Asahikawa Medical College, Asahikawa, Japan) for his helpful advice regarding the performance of this study and Y. Kobatake (Osaka City University, Osaka, Japan) for adenovirus preparation and purification. We also thank Dr. E. R. Stanley (Albert Einstein College of Medicine, New York, NY) for the Bac1.2F5 cells, Drs. I. Saito and Y. Kanegae (University of Tokyo, Tokyo, Japan) for the adenovirus transfer vectors (pAxCAwt and pAxcw), Dr. M. Weller (University of Tübingen, Tübingen, Germany) for the rat MCP-1 plasmid, Dr. J. Miyazaki (University of Osaka, 0saka, Japan) for the CAG promoter, and Dr. M. Miyagishi (University of Tokyo, Tokyo, Japan) for the pcPURmU6 plasmid and selection of the siRNA sequences. We are also grateful to T. Kawai for her secretarial assistance.

Correspondence should be addressed to Dr. Hiroshi Kiyama, Department of Anatomy and Neurobiology, Osaka City University Graduate School of Medicine, 1-4-3 Asahimachi, Abenoku, Osaka 545-8585, Japan. E-mail: kiyama@med.osaka-cu.ac.jp.

DOI:10.1523/JNEUROSCI.0023-06.2006

Copyright $\odot 2006$ Society for Neuroscience $\quad$ 0270-6474/06/267460-08\$15.00/0 phage influx into injured nerves are not fully understood, it is widely accepted that disruption of axon-Schwann cell contacts during axon degeneration triggers dedifferentiation of the Schwann cells and subsequently induces the cells to release mediators for macrophage attraction. A recent study suggested that secreted molecules from cultured Schwann cells or sciatic nerve explants are the most potent candidates for macrophage attractants (Tofaris et al., 2002). Among these factors, monocyte chemoattractant protein-1 (MCP-1) and leukemia inhibitory factor (LIF), released from Schwann cells after nerve injury, have been shown to have chemotactic activity for macrophages (Sugiura et al., 2000; Tofaris et al., 2002). However, knock-out mice of LIF or the MCP-1 receptor gene CCR2 (for the CC motif chemokine receptor 2) only show partial reduction of the macrophage recruitment to degenerating sciatic nerves (Siebert et al., 2000; Sugiura et al., 2000), strongly suggesting the existence of additional factors that play similar or even higher roles as macrophage chemoattractants during nerve regeneration.

Recently, we reported that pancreatitis-associated protein (PAP)-III, a member of the PAP/regenerating gene (Reg) family, is predominantly induced in the Schwann cells of injured nerves (Namikawa et al., 2005). The PAP/Reg family consists of secretory molecules, and much attention has been paid recently to their significant expressions in human cancer cells (Zhang et al., 2003). Among the family members, Reg-2/PAP-I [also known as HIP (hepatocarcinoma-intestine-pancreas)/PAP in humans (Zhang et al., 2003)] is the only member whose functions in the nervous system have been elucidated. Reg-2/PAP-I is massively upregulated in motor and sensory neurons after axotomy and was found to enhance nerve regeneration via stimulation of Schwann cell growth (Livesey et al., 1997). In addition, Reg-2/ 
PAP-I also functions as a survival factor for cultured motor neurons (Nishimune et al., 2000). In contrast to such remarkable functions of Reg-2/PAP-I released from injured neurons, the physiological relevance of PAP-III in injured nerves as well as other systems has hardly been investigated. Therefore, we examined the physiological relevance of PAP-III during nerve regeneration in the present study. Several aspects of the results obtained from our in vitro and in vivo experiments clearly indicate that PAP-III is a new member of the macrophage chemoattractants and is particularly involved in nerve regeneration.

\section{Materials and Methods}

Transwell assay using modified Boyden chambers. Proteose peptone (Difco, Detroit, MI)-elicited macrophages were collected from rat peritoneal cavities as described previously (Tofaris et al., 2002). A mouse macrophage cell line, Bac1.2F5, established by Dr. E. Richard Stanley's laboratory (Albert Einstein College of Medicine, New York, NY), was maintained according to their protocol (Morgan et al., 1987). First, 700 $\mu l$ of RPMI-1640 medium (Sigma, Tokyo, Japan) containing recombinant rat PAP-III (supplemental Method 1, available at www.jneurosci.org as supplemental material) or rat MCP-1 (Chemicon, Temecula, CA) or no factor was placed in the lower chambers. Cells were resuspended at a concentration of $1 \times 10^{5}$ cells $/ \mathrm{ml}$ (peritoneal macrophages) or $2.5 \times 10^{5}$ cells $/ \mathrm{ml}$ (Bac1.2F5 cells) in RPMI-1640 containing $0.1 \%$ BSA. Next, $200 \mu \mathrm{l}$ aliquots of the cell suspensions were placed in FALCON-HTS Fluoro Block inserts $(3.0 \mu \mathrm{m}$ pore size for peritoneal macrophages or $8.0 \mu \mathrm{m}$ pore size for Bac1.2F5 cells; BD Falcon, Bedford, $\mathrm{MA}$ ). At $2 \mathrm{~h}$ (for peritoneal macrophages) or $8 \mathrm{~h}$ (for Bac1.2F5 cells) after seeding, the cells migrating to the lower chambers were labeled with the fluorescent dye Calcein-AM (Invitrogen, Carlsbad, CA) according to the protocol of the manufacturer, and the numbers of migrated cells were counted after capturing at least five random $200 \times$ fields per well. At each point, six independent wells were used to determine the mean number of migrating cells. Data were calculated as the ratio of the mean number of migrated cells treated with PAP-III or MCP-1 relative to the control cells $(n=6)$.

Scratch assay. A wound was made in confluent monolayers of Bac1.2F5 cells grown on 12-well cell culture plates by scraping with a sterile $200 \mu \mathrm{l}$ Gilson (Middletown, WI) pipette tip. Next, the cells were rinsed with PBS and further cultured in DMEM (Sigma) supplemented with 10\% fetal calf serum (FCS) in the presence ( $1 \mathrm{ng} / \mathrm{ml}$ each) or absence of PAP-III or MCP-1. Using a CCD camera system, pictures of the cultures were taken immediately after scratching and then after 16 and $24 \mathrm{~h}$. The migration distances of the macrophages were measured according to a previous report (John et al., 2004) $(n=6)$.

Generation of adenoviral vectors and viral infections. Adenovirus vectors were constructed using a Takara (Otsu, Japan) adenovirus kit according to the protocol of the manufacturer. The detailed methods used for virus construction and viral infection of COS7 cells or sciatic nerves are described in supplemental Method 2 (available at www.jneurosci.org as supplemental material).

Animals and surgery. Experiments using animals were performed in accordance with the guidelines laid down by Osaka City University Medical School regarding the care and use of animals for experimental procedures. Adult male Wistar rats weighing 150-200 g were used throughout this study after anesthesia with pentobarbital. Their right sciatic nerves were axotomized or crushed. Some rats were operated on at $1 \mathrm{~d}$ after adenovirus infection. For nerve crush injury, the nerve was crushed with a pair of jeweler's forceps for $30 \mathrm{~s}$, and the crush site was tagged with an 8-0 Dermalon suture to create a mark on the injury site.

Chemotaxis assay in collagen gels. To evaluate the chemotactic activity of PAP-III, aggregates of Bacl.2F5 macrophages were embedded in collagen gels together with aggregates of green fluorescent protein (GFP)- or PAP-III-expressing COS1 aggregates as shown in Figure 3. GFP-, PAPIII-, or MCP-1-expressing adenoviruses were infected into COS1 cells as described above, followed by creation of aggregates of these cells by the hanging-drop method (Fan and Tessier-Lavigne, 1994) at $24 \mathrm{~h}$ after the adenovirus infection. Conversely, macrophage aggregates were created by placing single drops of a macrophage cell suspension $\left(6 \times 10^{4}\right.$ cells in $10 \mu$ l of DMEM) on plastic Petri dishes (BD Falcon), followed by incubation in a $\mathrm{CO}_{2}$ incubator for 3-4 h. After formation of the macrophage aggregates, COS1 aggregates were placed at $500 \mu \mathrm{m}$ from the edge of a macrophage aggregate, embedded in collagen gels $(8: 1: 1$ of $0.3 \%$ collagen/0.23 M NaOH-0.14 M NaHCO $/ 10 \times$ DMEM), and cultured in DMEM (Sigma) containing 10\% FCS. After $48 \mathrm{~h}$ of coculture, the aggregates were fixed with $4 \%$ paraformaldehyde and reacted with the F $4 / 80$ monoclonal antibody (1:200; Serotec, Raleigh, NC), a specific marker for mouse macrophages. An Alexa 594-conjugated anti-rat IgG (Invitrogen) was used as the secondary antibody, followed by staining with Hoechst 33258 (Wako, Osaka, Japan). Photographs of the cells were obtained using a CCD camera before and after each assay and saved on a computer. Next, the two photographs were merged to identify the edge of each macrophage aggregate before the migration, and the chemotactic index was determined as described in Fig. 3D. Conversely, nerves infected with adenoviruses expressing either siGFP [small interfering RNA (siRNA) sequence targeted against rat GFP mRNA] or siPAPIII (siRNA sequence targeted against rat PAP-III mRNA) as described above were sampled at $1 \mathrm{~d}$ after the axotomy. Nerve explants were cut as 3-mm-long segments, spanning from 2 to $5 \mathrm{~mm}$ distal from the injury site. The explants were then cocultured with a macrophage aggregate with the $2 \mathrm{~mm}$ end of the explant oriented toward the macrophage aggregate. The chemotactic index of each nerve was also estimated similar to that of COS1 aggregates $(n=8)$.

Reverse transcription-PCR. For mRNA detection in injured nerves, rats were killed at $1 \mathrm{~d}$ after sciatic nerve transection or crush injury. Total RNA was prepared from normal nerves and distal segments of the injured nerves $(0-5 \mathrm{~mm}$ from the injury site). Reverse transcription (RT)-PCR analysis was performed as described in supplemental Method 3 (available at www.jneurosci.org as supplemental material).

Western blotting. At $36 \mathrm{~h}$ after adenovirus infection, Western blotting analysis of COS7 cell lysates was performed as described previously (Namikawa et al., 2005). The following primary antibodies were used as probes: anti-PAP-III antibody (1:1000) (Namikawa et al., 2005) and anti-glyceraldehyde-3-phosphate dehydrogenase (GAPDH) monoclonal antibody (1:10,000; Ambion, Austin, TX). The reactions were visualized using an ECL system (GE Healthcare, Piscataway, NJ).

In vivo assay for macrophage recruitment and nerve regeneration. At $4 \mathrm{~d}$ (for macrophage and axon staining) or $7 \mathrm{~d}$ (for axon staining) after the nerve crush injury, adenovirus-infected sciatic nerves were removed and frozen in a block of Tissue Tek OCT. Serial $8 \mu \mathrm{m}$ longitudinal cryostat sections were cut and subjected to immunohistochemical staining as described previously (Dailey et al., 1998). ED-1 (1:5000; Serotec) or 2H3 (1:50; cell supernatant of $2 \mathrm{H} 3$ mouse hybridoma; Developmental Studies Hybridoma Bank, University of Iowa, Iowa City, IA) antibodies were used as primary antibodies for staining the macrophages or regenerating axons, respectively. Macrophage counts were performed by counting the numbers of ED-1-positive cells in areas of $0.04 \mathrm{~mm}^{2}$ at 1.5 and $3 \mathrm{~mm}$ from the crush site. Axon counts were also performed by counting regenerating axons stained with the $2 \mathrm{H} 3$ antibody in five fields at 3 and 6 $\mathrm{mm}$ from the crush site. The detailed protocols for the macrophage and axon counts are described in supplemental Method 4 (available at www.jneurosci.org as supplemental material).

Statistical analysis. The mean of the collected data were determined for each time point and experimental group. The statistical significance of differences in the counts between two groups was tested by Student's $t$ test. Statistical significance was set at $p<0.05$.

\section{Results \\ PAP-III stimulates migration of macrophages in Transwell assays}

Our previous report revealed that PAP-III expression is prominently upregulated in the distal portion of injured hypoglossal nerves and that this upregulation occurs rapidly within $12 \mathrm{~h}$ to reach a peak level at $1 \mathrm{~d}$ after axotomy (Namikawa et al., 2005). A rapid upregulation of PAP-III expression was also observed at $1 \mathrm{~d}$ after injury in our sciatic nerve injury model (supplemental Fig. 
$1 A$, available at www.jneurosci.org as supplemental material). In addition, PAP-III may be a secretory molecule, because PAP-III-transfected HEK293T cells secreted abundant PAP-III into the culture medium (supplemental Fig. $1 B$, available at www.jneurosci.org as supplemental material). Such a rapid gene expression profile and the secretory nature after injury are reminiscent of chemoattractants for macrophages. To examine the possibility that PAP-III acts as a macrophage chemoattractant, we first used one of the Transwell assays, known as the "modified Boyden chamber assay," which enables us to count fluorescently labeled macrophages migrating across a fluorescenceblocking planar micropore membrane (Yamakawa et al., 2000). Two sources of macrophages, namely rat peritoneal primary macrophages and the mouse bone marrow-derived cell line Bac1.2F5 (Morgan et al., 1987), were used for these assays. Recombinant rat PAP-III prepared using a yeast expression system (supplemental Fig. $1 B$, available at www.jneurosci.org as supplemental material) was added to the medium in the lower chambers at concentrations ranging from 0.01 to $500 \mathrm{ng} / \mathrm{ml}$, whereas cells were seeded into the upper chambers. Next, the cell migration to the lower chambers was measured. MCP-1 at $10 \mathrm{ng} / \mathrm{ml}$ was applied to the lower chambers for control experiments, because it has been shown previously to induce successful macrophage migration in Boyden chamber assays (Tofaris et al., 2002). In the experiments using primary macrophages and Bac1.2F5 cells, the numbers of migrated cells (arrowheads) in the lower chambers containing PAP-III or MCP-1 were significantly higher than those in control chambers without these factors (Fig. $1 A$ ). The most effective migration activities were induced by PAP-III at concentrations from 1 to 10 $\mathrm{ng} / \mathrm{ml}$. These results indicate that PAP-III promotes macrophage migration, similar to MCP-1. Furthermore, its activity was similar to that of MCP-1 for peritoneal macrophages and even higher for Bac1.2F5 cells (Fig. 1B).

\section{PAP-III enhances chemokinesis of macrophages in} scratch assays

Previous studies have argued that Transwell chamber assays represent a simple method for estimating cell migration but are not ideal for measuring chemoattraction (Zhu et al., 2002), because stimulation of cell motility (chemokinesis) could be sufficient to increase the number of migrating cells into the lower chambers. Thus, we performed additional experiments to determine whether the increase in migration observed in the Transwell assays was attributable to the activation of chemokinesis, chemotaxis, or both. First, to evaluate whether PAP-III induces chemokinesis of macrophages, we performed scratch assays as described in a previous study (John et al., 2004). A cell-free area was gener- ated by gently scratching a confluent cell monolayer with a pipette tip, resulting in the formation of a gap. Immediately after creation of the gap, PAP-III or MCP-1 was added to the medium, and the migration distances of the cells from the edges of the gaps were measured at 16 and $24 \mathrm{~h}$ after the scratch. Control experiments were performed in parallel without the addition of a factor. We only used Bac1.2F5 macrophages in these assays because primary peritoneal macrophages hardly reached confluence in the culture dishes, even in the presence of a growth factor such as colony stimulating factor-1 (data not shown). The macrophages exposed to either PAP-III or MCP-1 clearly demonstrated rapid migration into the gaps compared with control assays without a factor at each time point (Fig. 2A,B). This effect was not attributable to stimulation of cell growth because none of the concentrations of PAP-III and MCP-1 used in these assays induced macrophage growth, even in the presence of serum (data not shown). In terms of their chemokinesis activities, no significant differences were observed between PAP-III and MCP-1 (Fig. 2B). These results demonstrate that PAP-III enhances chemokinesis of macrophages, in a manner similar to MCP-1. 


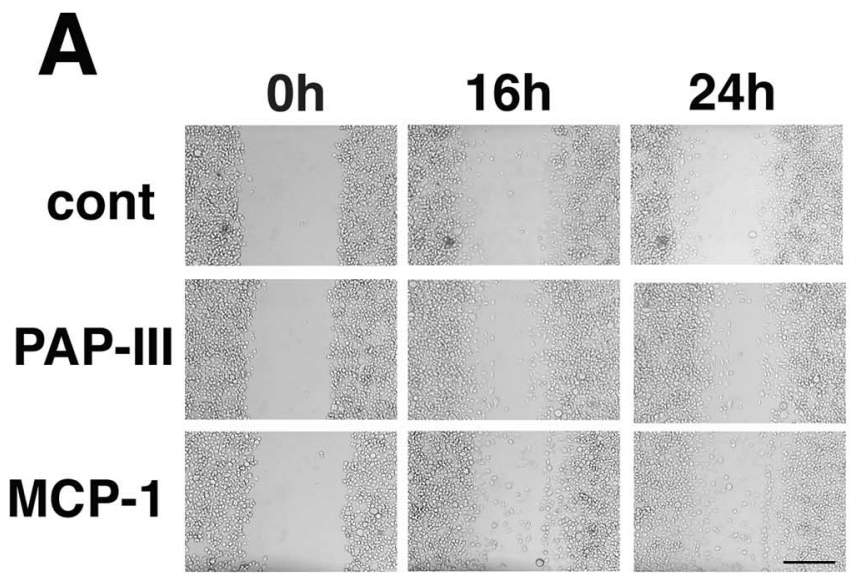

B

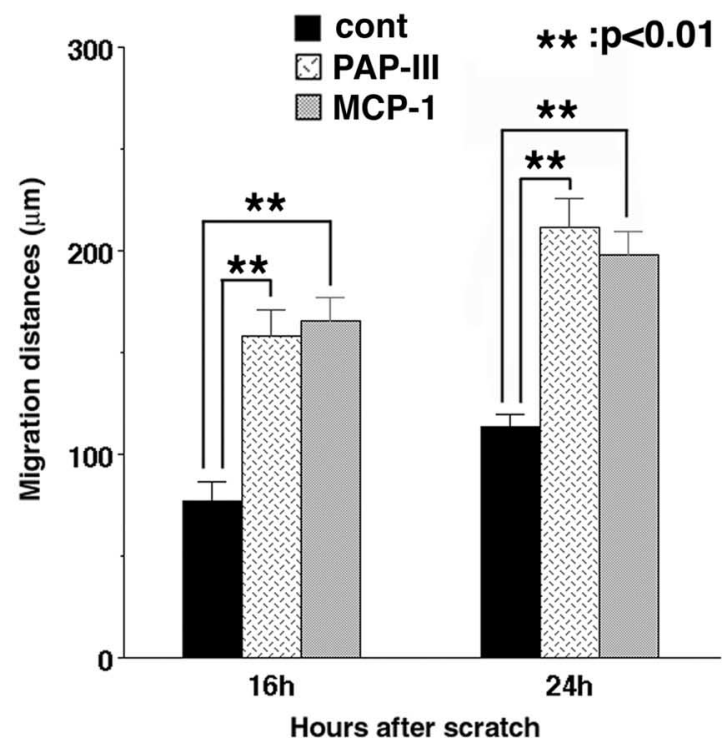

Figure 2. PAP-III promotes macrophage chemokinesis, as evaluated using scratch assays. Bac1.2F5 cells were grown to confluence on cell culture dishes, and then a gap was made using a sterile $200 \mu \mathrm{l}$ Gilson pipette tip in control cultures (cont) or cultures in the presence of PAP-III or MCP-1 at $1 \mathrm{ng} / \mathrm{ml}$. A, Photographs of the same fields were taken at 16 and $24 \mathrm{~h}$ after creation of the scratch. $\boldsymbol{B}$, The mean migration distances of the macrophages at each point after the scratch were measured. Error bars are the SEM. Scale bar, $500 \mu \mathrm{m}$.

PAP-III induces chemotaxis of macrophages in cocultures of aggregated macrophages and $\mathrm{COS}$ cells

Next, to further address the potential role of PAP-III as an activator of macrophage chemotaxis, we established a coculture system in collagen gels (Fig. 3). Aggregates of Bac1.2F5 cells attached to plastic dishes were cocultured and embedded in collagen gels with aggregates of COS cells expressing GFP, PAP-III, or MCP-1 using adenoviral gene transfers. PAP-III-expressing COS cells strongly attracted macrophages, which were positively stained by an anti-macrophage marker antibody (F4/80), whereas the GFPexpressing COS cells did not show a clear attraction (Fig. $3 A, C$ ). MCP-1-expressing aggregates also effectively attracted macrophages in this assay system (Fig. 3B). The numbers of cells migrating $>100 \mu \mathrm{m}$ toward to the center of the COS aggregates were counted, and the chemotactic index was calculated as the ratio of the migrating cells toward to each aggregate compared with that toward to the GFP-expressing aggregate (Fig. 3D). The
A

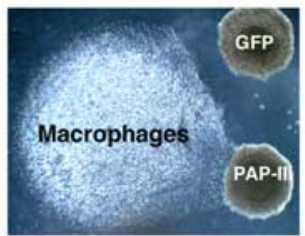

B

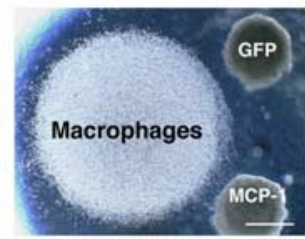

C

Hoechist

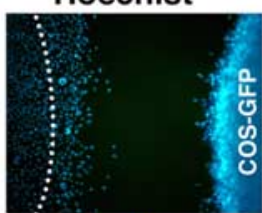

$\mathrm{F} 4 / 80$

GFP

PAP-III
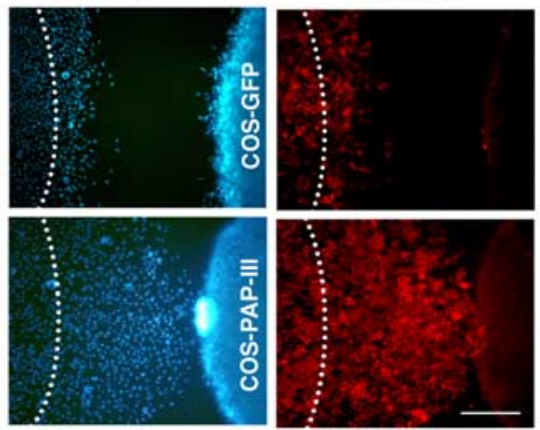

D
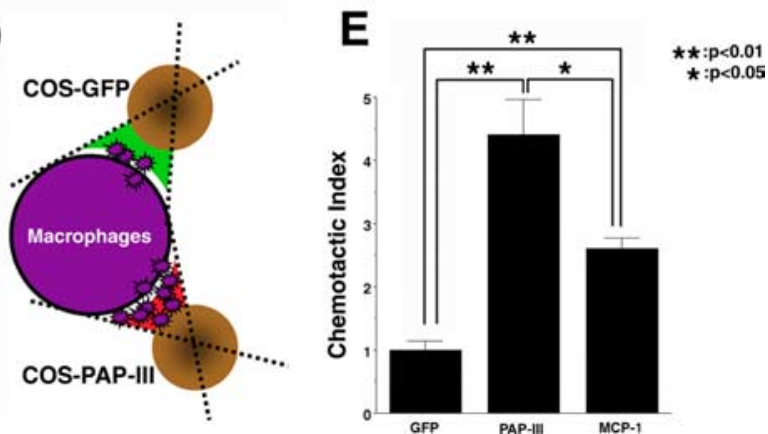

Figure 3. Cocultures of aggregated macrophages and COS cells reveal that PAP-III has a chemotactic activity for macrophages. $A, B$, Aggregates of $B a c 1.2 F 5$ macrophages and $C O S$ cells expressing GFP and either PAP-III ( $\boldsymbol{A}$ ) or MCP-1 (B) were embedded in collagen gels and cocultured for $48 \mathrm{~h}$. COS cell aggregates expressing PAP-III $(\boldsymbol{A})$ or MCP-1 $(\boldsymbol{B})$ strongly attract large numbers of macrophages. $C$, High-power magnification photographs showing that significant numbers of macrophages migrate toward a PAP-III-expressing aggregate (bottom) but not toward a GFP-expressing aggregate (top) after $48 \mathrm{~h}$ of coculture. The cells are visualized by either Hoechst staining (left column, blue) or F4/80 antibody staining (right column, red). The white dotted curve in each photograph indicates the edge of the macrophage aggregate when the assay started. $\boldsymbol{D}$, Evaluation of the chemotactic activities in these assays. The numbers of cells migrating $>100 \mu \mathrm{m}$ from the macrophage aggregate toward GFP-expressing COS cells (green field) and PAP-III-expressing COS cells (red field) were counted in each assay, and the chemotactic indexes were calculated as ratios of the number of migrating cells toward the PAP-III-expressing aggregate relative to the GFP-expressing aggregate. $E$, Chemotactic indexes of the $C O S$ aggregates. Error bars are the SEM. Scale bars: $A, B, 2 \mathrm{~mm} ; C, 200 \mu \mathrm{m}$.

chemotactic activity of PAP-III was apparently stronger than that of GFP. In addition, its activity was significantly higher than that of MCP-1 (Fig. 3E).

Knockdown of PAP-III gene expression in injured nerves suppresses the chemotactic activity

Although PAP-III is induced in injured nerves and functions as a chemoattractant for macrophages, it remains unclear whether nerve injury-induced PAP-III is a major macrophage attractant among the known and unknown factors released from injured nerves. To address this question, we generated a PAP-III genesilencing construct expressing an siRNA (Miyagishi and Taira, 2004) and used it to specifically suppress PAP-III expression in injured sciatic nerves. This unique $19 \mathrm{bp}$ siRNA sequence tar- 
A

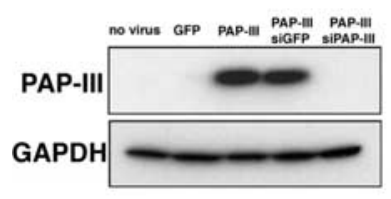

B

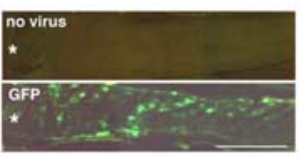

D

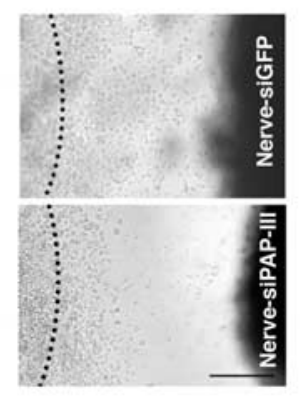

C

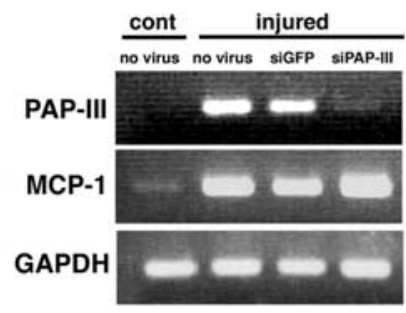

E

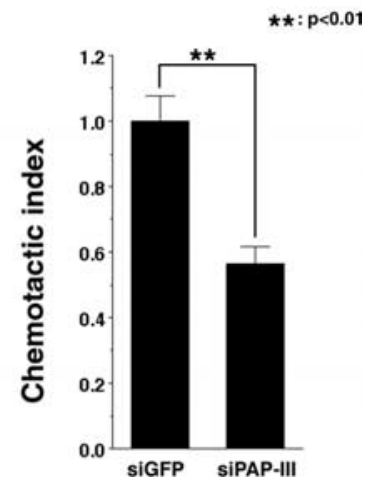

Figure 4. Knockdown of lesion-induced PAP-III expression leads to a reduction in its chemotactic activity of injured nerves. $A$, Western blotting using an anti-PAP-III antibody confirms that adenoviral expression of PAP-III in COS cells is clearly diminished by coinfection of an siPAP-IIIexpressing adenovirus, whereas coinfection of a control siGFP adenovirus has no effect. Equal amounts of protein loading were confirmed by Western blotting with an anti-GAPDH antibody. $\boldsymbol{B}$, Adenoviral gene transfer into sciatic nerves at $1 \mathrm{~d}$ after axotomy. Strong GFP expression is detected in the adenovirus-infected region (bottom, GFP), whereas no fluorescence signal was observed in control nerve $1 \mathrm{~d}$ after axotomy (top, no virus). Each asterisk indicates the nerve injury site. C, RT-PCR analysis using distal segments of injured sciatic nerves. Note that axotomyinduced PAP-III expression is effectively suppressed in siPAP-III-expressing nerves without changing the level of MCP-1 expression. Infection of a control siGFP-expressing adenovirus has no effect on the gene expression levels. The expression of GAPDH mRNA was used as an internal control. cont, Control. D, Photographs showing cocultures of macrophage aggregates with siRNA-expressing nerves (top, siGFP; bottom, siPAP-III) after $48 \mathrm{~h}$ embedded in collagen gels. The black dotted curve in each photograph indicates the edge of the macrophage aggregate when the assay started. $\boldsymbol{E}$, Evaluation of the chemotactic activity of each adenovirus-expressing nerve explant. Error bars are the SEM. Scale bars: $\boldsymbol{B}, 1 \mathrm{~mm} ; \boldsymbol{D}, 200 \mu \mathrm{m}$.

geted against rat PAP-III mRNA (siPAP-III) was cloned under the mouse U6 promoter. Plasmid vectors expressing siPAP-III and PAP-III were cotransfected into COS cells, and successful specific suppression of PAP-III protein expression was confirmed (supplemental Fig. 2, available at www.jneurosci.org as supplemental material). Next, adenoviruses expressing either siPAP-III or a control siRNA targeted against GFP mRNA (siGFP) were constructed to suppress PAP-III or for use as a control adenovirus in injured nerves, respectively. Coinfection of the PAP-III- and siPAP-III-expressing adenoviruses into COS cells successfully diminished the PAP-III expression, whereas the control siGFP had no effect on the level of PAP-III (Fig. 4A). According to a previously described optimized protocol for adenoviral gene transfer into injured nerves with higher efficiency (Joung et al., 2000), an intraneural adenovirus injection was performed into normal nerves of rats, and the axotomy was performed at $1 \mathrm{~d}$ after the infection. When the GFP-expressing adenovirus was injected using this procedure, strong GFP labeling of cells, possibly Schwann cells, was observed in injured nerves throughout the injected site at $1 \mathrm{~d}$ after the axotomy (Fig. $4 B$ ). Using this method, the endogenous PAP-III mRNA expression

was significantly reduced in siPAP-III-expressing nerves without changing the expression of MCP-1, whereas siGFP expression did not affect the expressions of either PAP-III or MCP-1 (Fig. 4C). The distal nerve segments of the injured nerves infected with the siRNA-expressing adenoviruses were explanted into collagen gels together with macrophage aggregates, and their macrophage chemotactic activities were measured as described above (Fig. 4D). The siGFP-expressing explants, which expressed a higher level of endogenous PAP-III, showed apparent macrophage attraction (Fig. $4 D$ ). Conversely, the siPAP-III adenovirus-infected nerve fragments, whose PAP-III expression level was almost abolished, demonstrated $\sim 50 \%$ lower activity (Fig. $4 D, E$ ). These results indicate that injury-induced PAP-III is one of the major attractants in injured nerves.

\section{PAP-III promotes macrophage recruitment and nerve regeneration in rats}

To gain additional insights into the physiological significance of lesion-induced PAP-III in vivo, PAP-III gene expressions were manipulated in a crush model of rat sciatic nerves by adenoviral gene transfer. The infection of adenoviruses expressing either PAP-III or siPAP-III into the distal segments of the injured nerves successfully controlled the level of PAP-III expression in the nerves at $1 \mathrm{~d}$ after the nerve injury (Fig. 5A). As expected, injuryinduced MCP-1 mRNA expression was not altered by these adenovirus infections, and the control adenoviruses expressing GFP or siGFP had no effects on any of the gene expressions (Fig. 5A). First, longitudinal sections of the injured nerves were stained with the ED-1 antibody, which specifically recognizes rat CD68, a lysosomal/endosomal marker of macrophages, at $4 \mathrm{~d}$ after the crush injury. This sampling time was selected as optimal based on observations of significant increases in the numbers of macrophages in the injured nerves around this time point. In the area $1.5 \mathrm{~mm}$ distal to the crush site, a massive increase in macrophage invasion was observed in PAP-III-overexpressing nerves, whereas similar increases were not seen in the control nerves (GFP and siGFP) (Fig. 5B,C). In the injured nerves expressing siPAP-III, the number of infiltrating macrophages was significantly lower than in the control nerves (Fig. $5 B, C$ ). Furthermore, substantial increases of macrophage invasion in PAP-IIIoverexpressing nerves were seen in areas $3 \mathrm{~mm}$ distal to the crush site in which macrophage influx was not fully achieved in control nerves (Fig. 5C).

Finally, to evaluate the effects of PAP-III on nerve regeneration, longitudinal sections of regenerating axons in the distal parts of injured nerves were stained with an anti-neurofilament (NF) antibody (Fig. 6). At $4 \mathrm{~d}$ after the crush injury, the regenerating nerve length appeared shorter in siPAP-III-expressing nerves than in siGFP-expressing nerves (Fig. 6A). Next, we measured the numbers of regenerating axons stained by the anti-NF antibody at 3 and $6 \mathrm{~mm}$ distal from the injury site in the nerves. PAP-III-knockdown nerves showed an $\sim 50 \%$ reduction in the number of regenerating axons compared with control siGFPexpressing nerves at $3 \mathrm{~mm}$ from the crush site and almost no NF-staining axons at $6 \mathrm{~mm}$ from the crush site. In contrast, several regenerating axons were counted in control nerves (Fig. $6 B, C)$. Furthermore, in PAP-III-overexpressing nerves, NFpositive axons extended farther from the crush site than those in control GFP-expressing nerves (Fig. 6A-C). A substantial increase in the number of regenerating axons was observed in PAPIII-overexpressing nerves. Specifically, $\sim 1$.6- and 2.7-fold increases were observed at 3 and $6 \mathrm{~mm}$ from the injury site, respectively, compared with the numbers in GFP-expressing 


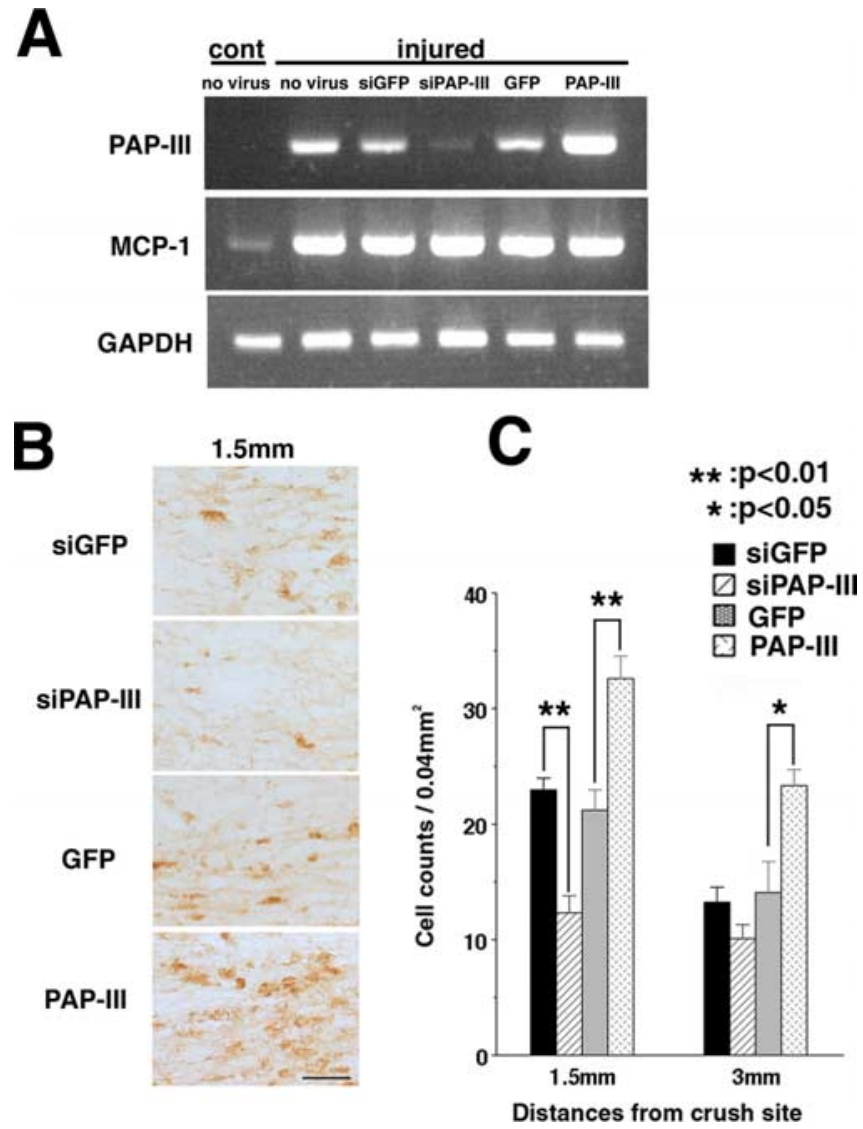

Figure 5. PAP-III expression regulates the chemoattractant activity for macrophages in degenerating nerves in vivo. $\boldsymbol{A}$, RT-PCR analysis showing that injury-induced PAP-III mRNA expression is enhanced or reduced by adenoviral gene transfer of PAP-III or siPAP-III, respectively, at $1 \mathrm{~d}$ after sciatic nerve crush injury. cont, Control. $\boldsymbol{B}$, The recruited macrophages in the degenerating nerves were stained with the ED- 1 antibody at $1.5 \mathrm{~mm}$ from the crush site at $4 \mathrm{~d}$ after the injury. Note that a higher number of macrophages is present in PAP-III-overexpressing nerves compared with GFP-expressing nerves. Conversely, a lower number of macrophages is observed in siPAP-III-expressing nerves than in control siGFP-expressing nerves. $\boldsymbol{C}$, Mean numbers of macrophages per $0.04 \mathrm{~mm}^{2}$ in longitudinal nerve sections at 1.5 and $3 \mathrm{~mm}$ distal to the crush site. Error bars are the SEM. Scale bar, $50 \mu \mathrm{m}$.

nerves (Fig. 6C). Similar tendencies for axon counts were observed in PAP-III gene-manipulated nerves $7 \mathrm{~d}$ after nerve injury (Fig. 6C). PAP-III would not directly function on Schwann cell or neuron, because primary cultures of Schwann cells in the presence of PAP-III could not show any significant cell growth. Furthermore, PAP-III failed to stimulate neurite elongation in organ-cultured dorsal root ganglion (supplemental Figs. 3, 4, available at www.jneurosci.org as supplemental material). Collectively, these in vivo results suggest that injured nerve-derived PAP-III is one of the crucial factors for macrophage attraction and perhaps subsequent peripheral nerve regeneration.

\section{Discussion}

In this study, we revealed physiological functions of PAP-III for the first time. PAP-III was found to stimulate both chemokinesis and chemotaxis of macrophages, as evaluated using three distinct assay systems in vitro, and these novel functions of PAP-III were further shown to be involved in peripheral nerve regeneration in vivo.

Macrophage recruitment by injured nerves is a prominent phenomenon for peripheral nerve regeneration. In injured nerves, infiltration of neutrophils is hardly observed, whereas a substantial influx of macrophages into degenerating nerves is seen at 2-3 d after nerve injury (Ramón y Cajal, 1928; Beuche and Friede, 1984; Perry et al., 1987; Stoll et al., 1989). These findings strongly suggest that macrophage chemoattractants are released from injured nerves, from either the injured nerve tips or Schwann cells. Among the factors released from injured nerves, MCP-1 from Schwann cells has been assumed to be the major macrophage chemoattractant (Tofaris et al., 2002), although faint activity of LIF has also been reported (Sugiura et al., 2000). However, application of a neutralizing antibody against MCP-1 to conditioned media from nerve segments and Schwann cell cultures does not completely reduce the chemotactic activity (Tofaris et al., 2002), suggesting the possibility that another chemoattractant is also released, presumably from injured Schwann cells. Our coculture system demonstrated that PAP-III has a greater chemotactic activity for macrophages than MCP-1, whereas cultured nerves whose PAP-III expression was knocked down without changing the MCP-1 expression showed greatly reduced chemotactic activity. These results clearly indicate that PAP-III could be the missing macrophage chemoattractant released from injured nerves.

The substantial induction of PAP-III in injured peripheral nerves is quite a specific response that has not been observed in other tissue injury models (data not shown), although acute pancreatitis can induce PAP-III mRNA expression together with the mRNAs of other Reg/PAP family members (Dusetti et al., 1995; Graf et al., 2002). In particular, we did not detect any induction of PAP-III expression in CNS glial cells, such as oligodendrocytes, or astrocytes in our spinal cord injury model (Suzuki et al., 2005). Conversely, MCP-1 is induced in a wide variety of traumatic damage and inflammation, including CNS injuries (Rollins, 1996; McTigue et al., 1998; Minami and Satoh, 2003). Actually, the accumulation of macrophages and/or microglial cells around an injury site is significantly delayed in the CNS (Perry et al., 1987; Perry and Gordon, 1991; Avellino et al., 1995), and subsequent removal of myelin from the injured CNS is also limited (George and Griffin, 1994; Avellino et al., 1995). Therefore, relatively specific induction of PAP-III expression and its strong chemotactic activity for macrophages would partially explain the reason why larger amounts of macrophages were capable of infiltrating degenerating peripheral nerves to facilitate nerve regeneration. In addition to the rapid accumulation of macrophages at an injury site in the PNS, a previous study suggested that their phagocytic capacity for myelin clearance is much higher in the PNS than in the CNS (Lazarov-Spiegler et al., 1996). In fact, exposure of monocytes to sciatic nerve segments, but not optic nerve segments, significantly enhances the phagocytic activity of these cells (Lazarov-Spiegler et al., 1996). Furthermore, transplantation of such monocytes stimulated by sciatic nerve segments can overcome CNS regeneration failure in optic nerves or spinal cord trauma to some extent (Lazarov-Spiegler et al., 1996; Rapalino et al., 1998). It remains unclear whether injury-induced PAP-III enhances such phagocytic activities in macrophages, and additional studies are required to reveal the PAP-III function in terms of myelin removal for successful nerve regeneration.

It is also possible that macrophages stimulated by PAP-III are able to secrete potent regeneration-promoting molecules. Accumulated macrophages after PNS injury, for example, produce Schwann cell migration factor (Horie et al., 2004) or proteases and their inhibitors that modify the extracellular matrix (La Fleur et al., 1996; Siebert et al., 2001). Macrophages can also release some factors that have beneficial effects on neuronal regrowth or 
survival, e.g., brain-derived neurotrophic factor (BDNF), interleukin (IL)-6, or glial cell line-derived neurotrophic factor (Batchelor et al., 1999; Dougherty et al., 2000; Leskovar et al., 2000). In fact, BDNF is especially shown to be involved in collateral branching from regenerating motor axons (Streppel et al., 2002). Therefore, macrophage accumulation in injured nerve may be associated with stimulation of the collateral sprouting of regenerated fibers as well as axon regrowing. Interestingly, Yin et al. (2003) revealed that activation of endogenous macrophages by intravitreal injections of Zymosan could facilitate optic nerve regeneration in vivo. Furthermore, they identified a novel factor released from activated macrophages that induces neurite outgrowth of retinal ganglion cells more strongly than other tropic factors in vitro (Yin et al., 2003). Another probable function of PAP-III is to suppress macrophage release of cytotoxic cytokines around a nerve injury site because another family member, Reg-2/PAP-I, was reported to have anti-inflammatory potential on macrophages in acute pancreatitis (Vasseur et al., 2004). Although all the above-mentioned functions of PAP-III require further elucidation, it is likely that PAP-III has other beneficial functions in addition to macrophage chemoattraction for facilitating nerve regeneration.

Although PAP-III has a potent macrophage chemoattractant activity, the intracellular signaling pathways stimulated by PAP-III remain to be elucidated. Regarding Reg-2/PAP-I, a few studies have reported that PKA, Akt, and NF $\kappa \mathrm{B}$ (Nishimune et al., 2000; Simon et al., 2003) are involved in the cell-protective effect mediated by Reg-2/PAP-I, although our preliminary experiments indicated that these molecules are not involved in the effects of PAP-III (data not shown). Regarding MCP-1, its receptor and intracellular signaling pathway have been identified (Rossi and Zlotnik, 2000; Jones et al., 2003). One of the G-protein-coupled receptors (GPCRs), CCR2, is a receptor for MCP-1, and a recent study revealed that phosphatidylinositol 3-kinase $\gamma(\mathrm{PI} 3 \mathrm{~K} \gamma)$ located downstream of this receptor is involved in the signaling of MCP-1 because macrophages lacking PI3K $\gamma$ show reduced migration and a lack of chemotaxis when stimulated by MCP-1 (Jones et al., 2003). PI3K $\gamma$, which transduces signals for actin cytoskeleton reorganization, has been shown to generate phosphatidylinositol-4, 5-biphosphate and/or phosphatidylinositol 3,4,5-trisphosphate at the leading edge of migrating cells via a GPCR in response to a chemoattractant gradient, and PI3K localization in such a restricted region of cells may be required for proper chemotaxis (Merlot and Firtel, 2003). In fact, the PAP-III-induced chemotaxis of macrophages in our coculture system was significantly suppressed in the presence of pertussis toxin, which inhibits $G_{i}$ signaling (data not shown).
This observation suggests that activation of the $\mathrm{G}_{\mathrm{i}}-\mathrm{PI} 3 \mathrm{~K} \gamma$ pathway may be involved in the activation of macrophage motility by PAP-III, although it remains elusive whether PAP-III can bind directly to a certain type of GPCRs to elicit the intracellular signaling.

In conclusion, we identified PAP-III as a novel macrophage chemoattractant released from Schwann cells, the expression of which is induced in response to nerve injury. Because the induction of PAP-III has a clear merit for the regeneration of injured peripheral nerves, it should be further examined whether PAP-III expression has similar benefits for CNS regeneration. Furthermore, macrophages activated by PAP-III may have additional advantages over those activated by MCP-1, and, in this respect, PAP-III may provide new therapeutic intervention strategies for various types of injuries and inflammatory diseases not only in the neural tissue but also in other peripheral tissues. 


\section{References}

Avellino AM, Hart D, Dailey AT, MacKinnon M, Ellegala D, Kliot M (1995) Differential macrophage responses in the peripheral and central nervous system during wallerian degeneration of axons. Exp Neurol 136:183-198.

Batchelor PE, Liberatore GT, Wong JY, Porritt MJ, Frerichs F, Donnan GA, Howells DW (1999) Activated macrophages and microglia induce dopaminergic sprouting in the injured striatum and express brain-derived neurotrophic factor and glial cell line-derived neurotrophic factor. J Neurosci 19:1708-1716.

Beuche W, Friede RL (1984) The role of non-resident cells in Wallerian degeneration. J Neurocytol 13:767-796.

Dailey AT, Avellino AM, Benthem L, Silver J, Kliot M (1998) Complement depletion reduces macrophage infiltration and activation during Wallerian degeneration and axonal regeneration. J Neurosci 18:6713-6722.

Dougherty KD, Dreyfus CF, Black IB (2000) Brain-derived neurotrophic factor in astrocytes, oligodendrocytes, and microglia/macrophages after spinal cord injury. Neurobiol Dis 7:574-585.

Dusetti NJ, Frigerio JM, Szpirer C, Dagorn JC, Iovanna JL (1995) Cloning, expression and chromosomal localization of the rat pancreatitisassociated protein III gene. Biochem J 307:9-16.

Fan CM, Tessier-Lavigne M (1994) Patterning of mammalian somites by surface ectoderm and notochord: evidence for sclerotome induction by a hedgehog homolog. Cell 79:1175-1186.

George R, Griffin JW (1994) Delayed macrophage responses and myelin clearance during Wallerian degeneration in the central nervous system: the dorsal radiculotomy model. Exp Neurol 129:225-236.

Graf R, Schiesser M, Lussi A, Went P, Scheele GA, Bimmler D (2002) Coordinate regulation of secretory stress proteins (PSP/reg, PAP I, PAP II, and PAP III) in the rat exocrine pancreas during experimental acute pancreatitis. J Surg Res 105:136-144.

Horie H, Kadoya T, Hikawa N, Sango K, Inoue H, Takeshita K, Asawa R, Hiroi T, Sato M, Yoshioka T, Ishikawa Y (2004) Oxidized galectin-1 stimulates macrophages to promote axonal regeneration in peripheral nerves after axotomy. J Neurosci 24:1873-1880.

Hughes PM, Perry VH (2000) The role of macrophages in degeneration and regeneration in the peripheral nervous system. In: Degeneration and regeneration in the nervous system (Saunders NR, Dziegielewska KM, eds), pp 263-283. Chichester, UK: Harwood Academic.

John GR, Chen L, Rivieccio MA, Melendez-Vasquez CV, Hartley A, Brosnan CF (2004) Interleukin- $1 \beta$ induces a reactive astroglial phenotype via deactivation of the Rho GTPase-Rock axis. J Neurosci 24:2837-2845.

Jones GE, Prigmore E, Calvez R, Hogan C, Dunn GA, Hirsch E, Wymann MP, Ridley AJ (2003) Requirement for PI 3-kinase gamma in macrophage migration to MCP-1 and CSF-1. Exp Cell Res 290:120-131.

Joung I, Kim HS, Hong JS, Kwon H, Kwon YK (2000) Effective gene transfer into regenerating sciatic nerves by adenoviral vectors: potentials for gene therapy of peripheral nerve injury. Mol Cell 10:540-545.

La Fleur M, Underwood JL, Rappolee DA, Werb Z (1996) Basement membrane and repair of injury to peripheral nerve: defining a potential role for macrophages, matrix metalloproteinases, and tissue inhibitor of metalloproteinases-1. J Exp Med 184:2311-2326.

Lazarov-Spiegler O, Solomon AS, Zeev-Brann AB, Hirschberg DL, Lavie V, Schwartz M (1996) Transplantation of activated macrophages overcomes central nervous system regrowth failure. FASEB J 10:1296-1302.

Leskovar A, Moriarty LJ, Turek JJ, Schoenlein IA, Borgens RB (2000) The macrophage in acute neural injury: changes in cell numbers over time and levels of cytokine production in mammalian central and peripheral nervous systems. J Exp Biol 203:1783-1795.

Livesey FJ, O’Brien JA, Li M, Smith AG, Murphy LJ, Hunt SP (1997) A Schwann cell mitogen accompanying regeneration of motor neurons. Nature 390:614-618.

McTigue DM, Tani M, Krivacic K, Chernosky A, Kelner GS, Maciejewski D, Maki R, Ransohoff RM, Stokes BT (1998) Selective chemokine mRNA accumulation in the rat spinal cord after contusion injury. J Neurosci Res 53:368-376.

Merlot S, Firtel RA (2003) Leading the way: directional sensing through phosphatidylinositol 3-kinase and other signaling pathways. J Cell Sci 116:3471-3478.

Minami M, Satoh M (2003) Chemokines and their receptors in the brain: pathophysiological roles in ischemic brain injury. Life Sci 74:321-327.

Miyagishi M, Taira K (2004) RNAi expression vectors in mammalian cells. Methods Mol Biol 252:483-491.
Morgan C, Pollard JW, Stanley ER (1987) Isolation and characterization of a cloned growth factor dependent macrophage cell line, BAC1.2F5. J Cell Physiol 130:420-427.

Namikawa K, Fukushima M, Murakami K, Suzuki A, Takasawa S, Okamoto H, Kiyama H (2005) Expression of Reg/PAP family members during motor nerve regeneration in rat. Biochem Biophys Res Commun 332:126-134.

Nishimune H, Vasseur S, Wiese S, Birling MC, Holtmann B, Sendtner M, Iovanna JL, Henderson CE (2000) Reg-2 is a motoneuron neurotrophic factor and a signalling intermediate in the CNTF survival pathway. Nat Cell Biol 2:906-914.

Perry VH, Gordon S (1991) Macrophages and the nervous system. Int Rev Cytol 125:203-244.

Perry VH, Brown MC, Gordon S (1987) The macrophage response to central and peripheral nerve injury. A possible role for macrophages in regeneration. J Exp Med 165:1218-1223.

Ramón y Cajal S (1928) Degeneration and regeneration of the nervous system. London: Oxford UP.

Rapalino O, Lazarov-Spiegler O, Agranov E, Velan GJ, Yoles E, Fraidakis M, Solomon A, Gepstein R, Katz A, Belkin M, Hadani M, Schwartz M (1998) Implantation of stimulated homologous macrophages results in partial recovery of paraplegic rats. Nat Med 4:814-821.

Rollins BJ (1996) Monocyte chemoattractant protein 1: a potential regulator of monocyte recruitment in inflammatory disease. Mol Med Today 2:198-204.

Rossi D, Zlotnik A (2000) The biology of chemokines and their receptors. Annu Rev Immunol 18:217-242.

Scherer SS, Salzer JL (2001) Axon-Schwann cell interactions during peripheral nerve regeneration. In: Glial cell development, Ed 2 (Jessen KR, Richardson WD, eds), pp 299-317. London: Oxford UP.

Siebert H, Sachse A, Kuziel WA, Maeda N, Bruck W (2000) The chemokine receptor CCR2 is involved in macrophage recruitment to the injured peripheral nervous system. J Neuroimmunol 110:177-185.

Siebert H, Dippel N, Mader M, Weber F, Bruck W (2001) Matrix metalloproteinase expression and inhibition after sciatic nerve axotomy. J Neuropathol Exp Neurol 60:85-93.

Simon MT, Pauloin A, Normand G, Lieu HT, Mouly H, Pivert G, Carnot F, Tralhao JG, Brechot C, Christa L (2003) HIP/PAP stimulates liver regeneration after partial hepatectomy and combines mitogenic and antiapoptotic functions through the PKA signaling pathway. FASEB J 17:1441-1450.

Stoll G, Griffin JW, Li CY, Trapp BD (1989) Wallerian degeneration in the peripheral nervous system: participation of both Schwann cells and macrophages in myelin degradation. J Neurocytol 18:671-683.

Streppel M, Azzolin N, Dohm S, Guntinas-Lichius O, Haas C, Grothe C, Wevers A, Neiss WF, Angelov DN (2002) Focal application of neutralizing antibodies to soluble neurotrophic factors reduces collateral axonal branching after peripheral nerve lesion. Eur J Neurosci 15:1327-1342.

Sugiura S, Lahav R, Han J, Kou SY, Banner LR, de Pablo F, Patterson PH (2000) Leukaemia inhibitory factor is required for normal inflammatory responses to injury in the peripheral and central nervous systems in vivo and is chemotactic for macrophages in vitro. Eur J Neurosci 12:457-466.

Suzuki A, Namikawa K, Konishi H, Takaoka K, Kiyama H (2005) Expression of regenerating gene family members in response to CNS and PNS injuries. Soc Neurosci Abstr 31:102.9.

Tofaris GK, Patterson PH, Jessen KR, Mirsky R (2002) Denervated Schwann cells attract macrophages by secretion of leukemia inhibitory factor (LIF) and monocyte chemoattractant protein-1 in a process regulated by interleukin-6 and LIF. J Neurosci 22:6696-6703.

Vasseur S, Folch-Puy E, Hlouschek V, Garcia S, Fiedler F, Lerch MM, Dagorn JC, Closa D, Iovanna JL (2004) p8 improves pancreatic response to acute pancreatitis by enhancing the expression of the anti-inflammatory protein pancreatitis-associated protein I. J Biol Chem 279:7199-7207.

Yamakawa S, Furuyama Y, Oku N (2000) Development of a simple cell invasion assay system. Biol Pharm Bull 23:1264-1266.

Yin Y, Cui Q, Li Y, Irwin N, Fischer D, Harvey AR, Benowitz LI (2003) Macrophage-derived factors stimulate optic nerve regeneration. J Neurosci 23:2284-2293.

Zhang YW, Ding LS, Lai MD (2003) Reg gene family and human diseases. World J Gastroenterol 9:2635-2641.

Zhu Y, Yu T, Zhang XC, Nagasawa T, Wu JY, Rao Y (2002) Role of the chemokine SDF-1 as the meningeal attractant for embryonic cerebellar neurons. Nat Neurosci 5:719-720. 